suggests that phylotypes differ phenotypically. An accurate survey of genetic diversity and population structure will facilitate responsible selection of drug and/or vaccine targets for future treatments, and will enable better understanding of virulence factors contributing to the wide range of severity of symptoms associated with trichomoniasis.

\section{P4-S3.08 DEVELOPMENT OF A NOVEL CHIMERIC PROTEIN CONSTRUCT FOR IMPROVED DIAGNOSIS OF SYPHILIS}

doi:10.1136/sextrans-2011-050108.523

B Smith, C Cameron. University of Victoria, Victoria, Canada

Objectives To develop a novel diagnostic protein in order to enhance early detection of syphilis infections and improve overall syphilis diagnosis.

Methods Using pooled serum samples from patients infected with syphilis, immunoreactive regions of two previously identified diagnostic protein candidates, Tp0326 and Tp0453, were elucidated. Focusing on these regions, a chimeric protein construct was created for expression in Escherichia coli and expression conditions were optimised to produce soluble protein expression. This Tp0326/Tp0453 chimeric construct was screened against serum samples from; patients with primary, secondary, latent, and neurosyphilis and uninfected individuals. These results were directly compared to the rapid plasma regain (RPR) test, and the microhemagglutination assay for $T$ pallidum (MHA-TP).

Results Screening results show high degrees of sensitivity and specificity for the Tp0326/Tp0453 chimeric construct, identifying all stages of syphilis infection from early primary to late latent.

Conclusion The Tp0326/Tp0453 chimera shows promise as a new diagnostic antigen for detecting all stages of syphilis infection and for development into point-of-care diagnostic test formats.

\section{P4-S3.09 THE PREVALENCE OF TRICHOMONAS VAGINALIS VIRUS (TVV) IN GLOBALLY DISTRIBUTED TRICHOMONAS VAGINALIS ISOLATES}

doi:10.1136/sextrans-2011-050108.524

L Lezeau, M Conrad, A Gorman, J Carlton. New York University School of Medicine, New York, USA

Objective Trichomonas vaginalis, a highly prevalent non-viral sexually transmitted infection, has been shown to be infected by a doublestranded RNA virus known as T vaginalis virus (TVV). The presence of this virus has been associated with increased trafficking of the immunogenic P270 to the surface of the parasite, and has therefore been hypothesised to be an important virulence factor in trichomoniasis. In the present study, we investigate the prevalence of TVV in globally distributed $T$ vaginalis isolates and find an association between TVV infection and genetically distinct $T$ vaginalis populations.

Methods $150 T$ vaginalis isolates from the USA, Mexico, Italy, Southern Africa, Papua New Guinea and Australia were screened for TVV infection by running total RNA extract on $1 \%$ agarose gels to detect the presence or absence of the diagnostic $4.5 \mathrm{~kb}$ dsRNA genome of the virus. The prevalence of TVV in genetically distinct $T$ vaginalis phylotypes was compared using $\chi^{2}$ tests.

Results TVV was found to be present in $37 \%$ of $T$ vaginalis isolates. We find a difference in the prevalence of TVV infection between genetically distinct populations of parasites, with $3 \%$ of phylotype 1 isolates containing the virus vs $73 \%$ of phylotype 2 parasites $(<0.001)$. Conclusions TVV prevalence varies between $T$ vaginalis phylotypes 1 and 2. This finding has implications suggesting that TVV is transmitted vertically among parasites, as more closely related $T$ vaginalis strains are infected with TVV. Preliminary data also suggests that phylotype 2 parasites may show greater virulence, and further studies will be required in determining the role of TVV in this increased pathology.

\section{P4-S3.10 A TRICHOMONAS VAGINALIS HUMAN VACCINE CANDIDATE: FREUND'S ADJUVANT VS ALUMINIUM HYDROXIDE ADJUVANT IN A BALB/C MOUSE VAGINAL INFECTION MODEL}

doi:10.1136/sextrans-2011-050108.525

1J Smith, ${ }^{2,3} \mathrm{G}$ Garber. ${ }^{1}$ University of Ottawa, Ottawa, Canada; ${ }^{2}$ University of Ottawa, Faculty of Medicine, Canada; ${ }^{3} \mathrm{OHRI}$ Division of Infectious Disease, Canada

Background Trichomonas vaginalis is an underreported STD known to increase HIV transmission. Development of a vaccine against $T$ vaginalis could greatly affect transmission of $T$ vaginalis and HIV as well as reducing their global prevalence. Herein, we aim to investigate the feasibility of developing a $T$ vaginalis vaccine using the human-safe adjuvant Alhydrogel compared against an already established Freund's adjuvanted vaccine in a BALB/c vaginal infection mouse model.

Methods A prime-boost vaccination schedule was employed with live, whole cell $T$ vaginalis $(1 \times 107 \mathrm{Tv} / \mathrm{ml})$ is injected in $2-200 \mathrm{ul}$ aliquots 4 weeks apart and prior to vaginal infection challenge. Either Freund's, $0.5 \mathrm{mg} \mathrm{Al} / \mathrm{ml}$ Alyhydrogel, or $0.75 \mathrm{mg} \mathrm{Al} / \mathrm{ml}$ Alhydrogel were applied as adjuvants. Additionally, $0.75 \mathrm{mg} \mathrm{Al} / \mathrm{ml}$ Alhydrogel sham and non-vaccinated groups serve as controls. The $\mathrm{BALB} / \mathrm{c}$ vaginal environment was modified to mimic humans by adjustment of vaginal flora and estrogenisation prior to vaginal infection. The total IgG, IgG1 and IgG2a levels in serum were tested 3 weeks post each vaccine injection, then 2 and 4 weeks post-vaginal infection by ELISA. Groups were compared using Tukey's MCT (Post-Hoc one-way ANOVA; $\boldsymbol{\alpha}<0.05$ ).

Results Alhydrogel adjuvanted groups showed comparable total IgG and IgG1 levels to Freund's adjuvanted group. These levels for all adjuvanted groups were significantly different from control groups at all time points following boost-vaccination. IgG2a levels were not as consistent with large SD seen within Freund's adjuvanted mice. Alhydrogel adjuvanted groups were not significantly different from control groups at any time point for IgG2a levels suggesting no induction of IgG2. Controls and sham vaccines showed no Ig response. Conclusions Our data suggest that in line with expected Th2 skewed response from Alhydrogel adjuvant and Th1 skewed response from Freund's adjuvant there was a difference in IgG2a antibody production. Additionally, the Alhydrogel adjuvanted vaccines are otherwise similar to Freund's adjuvant in terms of total IgG and IgG1 levels suggesting the feasibility to pursue Alhydrogel as a vaccine candidate. Alhydrogel induces a significantly elevated immune response compared to natural infection antibody production (non-vaccinated control) and does not induce a nonspecific immune response.

\section{Basic sciences poster session 4: Bacterial diversity}

P4-S4.01 INVESTIGATION OF THE BACTERIAL DIVERSITY IN URINE
OF URETHRITIS PATIENTS AND HEALTHY CONTROLS
USING 454 HIGH-THROUGHPUT-SEQUENCING

doi:10.1136/sextrans-2011-050108.526

${ }^{1} \mathrm{P}$ Ahrens, ${ }^{1} \mathrm{M}$ Frølund, ${ }^{2} \mathrm{~W}$ A Al-Soud, ${ }^{3} \mathrm{~A}$ Wikström, ${ }^{3} \mathrm{P}$ Lidbrink, ${ }^{3} \mathrm{M}$ Cullberg, ${ }^{2} \mathrm{~S}$ J Sørensen, ${ }^{1} \mathrm{~J} S$ Jensen. ${ }^{1}$ Statens Serum Institut, Copenhagen, Denmark; ${ }^{2}$ University of Copenhagen, Denmark; ${ }^{3}$ Karolinska University Hospital, Sweden

Background Non-gonococcal urethritis (NGU) is a common sexually transmitted disease in men but in $30-50 \%$ of NGU cases, no known organism is found. We have used 454 high throughput sequencing to analyse the micro flora in urine samples from cases of urethritis and from controls. 
Methods Urine samples from men, 10 patients with urethritis and $>5$ $\mathrm{PMNL} / \mathrm{hpf}$ and 10 healthy controls with $<5 \mathrm{PMNL} / \mathrm{hpf}$, were collected. All samples were tested for Neisseria gonorrhoeae, Chlamydia trachomatis, Mycoplasma genitalium, Ureaplasma urealyticum, U parvum, Trichomonas vaginalis, Herpes Simplex Virus type 1 and 2 and Adenovirus using specific PCR assays. The V3 and V4 regions of the $16 \mathrm{~S}$ rRNA gene were PCR amplified, tagged and sequenced using the Titanium kit and GS FLX pyrose-quencing system (Roche) according to manufacturer's instructions. Sequences were analysed using the RDP Pyrosequencing Pipeline and CLC Genomics Workbench.

Results From each of the 20 samples, 8150 quality filtered sequences were randomly selected. Sequences were assigned to the genus level using the RDP Classifier. A total of 172 gen-era were identified, 133 in patients and 104 in controls. The median number of genera was 35.5 (19-49) in patients with urethritis compared to $25(16-43)$ in the controls. No single genus was present in all samples. Members of the genera Pseudomonas and Sphingomonas were present in all the controls and in most of the patient samples. Also, representatives of the genera Brevundimonas, Micrococcus, Bradyrhizobium and Chryseobacterium were present in 15-18 of the samples. Of the 172 genera, 77 were only found in a single sample and 33 were found in two different samples. Rarefaction analysis at the $5 \%$ level (comparable to genus) suggested that all the controls and most urethritis samples harboured each between 31 and 125 different groups. Four of the urethritis samples apparently contained between 150 and 300 different groups.

Conclusions Urine, even from healthy men, contains a very diverse micro-flora. Though not statistically significant, the total and median number of genera was found higher in patients with urethritis than in controls. Several widespread genera are likely to represent commensals and bacteria present in the environment.

\section{P4-S4.02 A 22-ORGANISM MICROARRAY APPROACH FOR DETECTING MICROBIOLOGICAL ASSOCIATIONS WITH SYMPTOMATIC URETHRITIS IN MALES}

doi:10.1136/sextrans-2011-050108.527

${ }^{1} S$ Patel, ${ }^{2} \mathrm{M}$ Pond. ${ }^{1}$ St George's Healthcare NHS Trust, London, UK; ${ }^{2}$ St George's, University of London, London, UK

Background In addition to its known microbiological aetiology, urethritis in men may be linked with other genital tract organisms, as yet unidentified in its pathogenesis. We used a microarray, with capacity to detect 22 genital tract organisms, in order to determine the association of symptomatic urethritis with infection or carriage of these organisms.

Methods 129 patients were asked to provide an extra first void urine specimen or give permission for their residual urine specimen submitted for Chlamydia trachomatis NAAT testing to be utilised. Patients were categorised into three self-reported symptom groups: definite symptoms of urethritis (discharge and/or dysuria), category 1 (C1) $n=80$; non-specific symptoms of urethritis (eg, minimal urethral discomfort), category 2 (C2) $n=26$; and asymptomatic category 3 (C3) $n=23$. Total urine nucleic acid was extracted and subsequently used for PCR coupled microarray analysis. Organisms were defined as present or absent using an online data analysis method. In a pre-planned analysis, the following categories were compared for prevalence of organisms: C1 vs C2 and C3 combined; C1 and C2 combined vs C3 using Fisher's exact test.

Results One or more organisms were detected in $74 \%(n=95)$ of patients and two or more organisms in $33 \%(n=42)$. The prevalence of organisms known to cause urethritis in this largely symptomatic cohort was: $16 \%(n=21), 9 \%(n=12)$ and $5 \%(n=6)$ for $C$ trachomatis, Mycoplasma genitalium and for Neisseria gonorrhoeae respectively. Escherichia coli was the most prevalent organism detected with a prevalence of $18 \%(n=23)$. The presence of $M$ genitalium was stat- istically associated with $\mathrm{C} 1$ and $\mathrm{C} 1$ and $\mathrm{C} 2$ combined ( $\mathrm{p}=0.03$ and 0.01 respectively). In symptomatic patients, $C$ trachomatis, Ureaplasma urealyticum, and Gardnerella vaginalis appeared to be more prevalent than in asymptomatics although not statistically significantly. Lactobacilli where detected in $1.3 \%$ and $4 \%$ of patients with C1 and C2 symptoms respectively, compared with $17 \%$ of asymptomatic patients. The absence of lactobacilli was associated with urethritis symptoms, either C1 alone or C1 and C2 combined $(p=0.03$ and $p=0.01$ ) respectively.

Conclusions Using a polymicrobial microarray approach we have demonstrated that symptomatic urethritis is associated with depletion of lactobacilli. This confirms early work using urethral swabs. The temporal nature of Lactobacilli depletion in relation to the onset of symptomatic urethritis needs to be investigated further.

\section{P4-S4.03 A LOW-COST MICROFLUIDICS-BASED DIAGNOSTIC TEST FOR STDs}

doi:10.1136/sextrans-2011-050108.528

\section{S Sia. Columbia University, New York, USA}

Background Undiagnosed and untreated STIs cause large morbidity and mortality, including birth defects and stillborn babies. Since most STI's have known treatments, the largest barriers for treating patients include high cost of transporting specimens to central labs and lack of access to diagnostic testing. We present data on a portable and low-cost microfluidics device for point-of-care diagnosis of multiple STDs (such as HIV and syphilis) in combination. In order to reduce the cost and size of the assay while maintaining high performance, we incorporated microfluidic designs such as single-use plastic microfluidic cassettes, a passive method for delivering reagents, and an amplification chemistry using gold nanoparticles.

Methods Our overall device is named mChip (mobile microfluidic chip for immunoassay on protein markers). We demonstrated an ability of mChip to simultaneously detect antibodies against HIV and syphilis in needle-pricked sample volumes. Both commercial specimens and archived specimens from Sub-Saharan Africa were used.

Results The test sensitivities and specificities for detection of HIVspecific antibodies and treponemal-specific antibodies matched the performances from lab-based ELISA. Compared to ELISA, our test can be performed anywhere, uses a very small volume of blood, and is about 10 times faster. Some of these results are in press in Nature Medicine, 2010.

Conclusion The mChip provided excellent performance in the diagnosis of HIV using only $1 \mu \mathrm{l}$ of unprocessed whole-blood and $<15$ min assay-time, and an ability to simultaneously diagnose HIV and syphilis with sensitivities and specificities equal to lab-based assays. Overall, we demonstrate an integrated strategy for miniaturising complex laboratory assays using microfluidics and nanoparticles to enable POC diagnostics and early detection of infectious diseases in remote settings.

\section{P4-S4.04 TEMPORAL DYNAMICS OF VAGINAL BACTERIAL COMMUNITIES}

doi:10.1136/sextrans-2011-050108.529

1J Ravel, 'R Brotman, ${ }^{1} \mathrm{P}$ Gajer, ${ }^{1} \mathrm{~J}$ Sakamoto, ${ }^{1} \mathrm{~S}$ Koenig, ${ }^{1} \mathrm{~L}$ Fu, ${ }^{2} \mathrm{X}$ Zhou, ${ }^{2} \mathrm{Z}$ Abdo, ${ }^{2} \mathrm{~L}$ Forney, 'J Ravel. 'University of Maryland, School of Medicine, Baltimore, USA; ${ }^{2}$ University of Idaho, USA

Background Dysbiosis of vaginal bacterial communities have been associated with increased risk for sexually transmitted infections and bacterial vaginosis. This is the first observational study to model temporal dynamics of vaginal microbiota using frequently collected samples, behavioural data and culture-independent methods. 